\title{
On the Rate of Convergence of an Alternating Direction Implicit Method in a Noncommutative Case*
}

\author{
By Olof B. Widlund
}

1. Introduction. It is well known that alternating direction implicit methods (ADI-methods) often provide very powerful techniques for computing numerical solutions to partial differential equations of elliptic type. The theoretical explanation for this is incomplete. The theory is in fact so unsatisfactory that it is often not possible to predict whether a suggested ADI-scheme is likely to converge or not.

Consider the following Dirichlet problem

$$
\begin{gathered}
-\frac{\partial}{\partial x_{1}}\left(a_{1}(x) \frac{\partial u}{\partial x_{1}}\right)-\frac{\partial}{\partial x_{\iota}}\left(a_{\llcorner}(x) \frac{\partial u}{\partial x_{2}}\right)+c(x) u=f(x), \quad x \in R ; \\
u(x)=g(x), \quad x \text { on the boundary of } R,
\end{gathered}
$$

where $x=\left(x_{1}, x_{2}\right)$ and $R$ is the interior of a compact region. The coefficients $a_{1}, a_{2}$ and $c$ are real valued sufficiently smooth functions. $a_{1}$ and $a_{2}$ are strictly positive and $c$ is bounded from below by a sufficiently large lower bound $c_{0}$. Let us introduce a rectangular lattice of mesh points on the region. We approximate the boundary of the region by nearby mesh points at which the solution is approximately known. If we set up the standard five point difference approximation to (1.1) on this lattice, we get a system of linear equations with as many equations as unknowns. It can be shown that this system has a unique solution if $c_{0}$ is sufficiently large. It is well known that if the number of unknowns is very large it is often best to solve such a system with some iterative method. In this paper we will consider an ADI-method which for the case $a_{1}=a_{2}=1$ and $c=0$ is identical with the original ADI-method of Peaceman and Rachford [3]. It is known both from existing theory and numerical experiments that this ADI-method, using a sequence of different iteration parameters, is often superior to other iterative methods. Cf. Birkhoff, Varga and Young [2]. In practice one usually chooses the parameters in a cyclic way. In this paper we will always suppose that this is done and we will call the number of parameters $m$ the cycle length. How to make the best choice for this number of steps, $m$, will not be discussed in this paper.

Before we describe our new result we will give a short survey of certain aspects of the theory for ADI-methods. For details we refer to Birkhoff, Varga and Young [2], Varga [5] and Wachspress [6].

Let us thus first consider Laplace's equation with Dirichlet data given on the boundary of a unit square. Suppose we use a uniform lattice of mesh points with

Received May 25, 1966.

* This paper represents results obtained at the Courant Institute of Mathematical Sciences, New York University. It was sponsored by the Swedish Technical Research Council and the Office of Naval Research, Contract Nonr-285(46).

Reproduction in whole or in part is permitted for any purpose of the United States Government. 
mesh size $h$. It is then possible, for any given $m$, to construct a set of iteration parameters for which the discrete $L^{2}$-norm of the error function is reduced by at least a factor

$$
p_{m}=\left(\frac{1-(\tan (\pi h / 2))^{1 / m}}{1+(\tan (\pi h / 2))^{1 / m}}\right)^{2}
$$

for each full cycle of iterations. For the same problem the spectral radius of the point successive over relaxation operator is equal to $p_{1}$. The number of arithmetic operations used to complete a step in the ADI-scheme is of the same order as for the successive over relaxation method. These facts explain why ADI-methods can be much more efficient than other iterative methods when $h$ is small and $m \geqq 2$. It has been shown that essentially the same rapid convergence is achieved in more general cases provided certain operators commute. Birkhoff and Varga [1] have however shown that these commutativity conditions impose very strong restrictions on both the coefficients and the region, which must then be rectangular.

Very little is known for noncommutative cases except when $m=1$. In this oneparameter case there exists a simple convergence proof which at the same time gives an adequate estimate for the asymptotic rate of convergence. However, as we have seen earlier, $m=1$ is the least interesting case. Some weak results have also been proved for the multi-parameter case. Thus Pearcy [4] has shown that we can always get convergence for any given problem and any given mesh if we choose $m$ sufficiently large, have a mild restriction on the size of the iteration parameters and use them in a certain order. Pearcy's technique is however not refined enough to give more than a very weak estimate for the rate of convergence. But it is known from numerical experiments that the same rapid convergence as in commutative cases is possible even in noncommutative cases.

In this paper we will consider equations of type (1.1) in rectangular regions. Our operators will then in general not commute. For any given $m$ we will construct iteration parameters which will make our scheme converge rapidly if the mesh size is sufficiently small. We will also be able to show that our estimate for the spectral radius of the operator which corresponds to a full cycle of iterations differs from that in a comparable commutative case only by a lower order term. Our technique would enable us to exhibit an estimate for this difference in terms of the mesh size, the iteration parameters, the coefficients and certain of their derivatives. Our iteration parameters will in general depend on the space variables. This dependence is however quite simple and it therefore adds little to the programming effort. Furthermore, a numerical experiment indicates that our theorems are not true if we restrict our choice to parameters which do not vary with the space variables. The result of this and other numerical experiments will be reported in a separate paper.

2. Preliminaries and Statement of Results. To simplify the writing up of our proofs we will suppose that our region is a unit square and that our lattice of mesh points is uniform, i.e. $\Delta x_{1}=\Delta x_{2}=h$. The extension of our results to rectangular regions and rectangular lattices is immediate. We suppose that $h=1 / N$ for some integer $N$ and that $h \leqq h_{0}$ where $h_{0}$ is a given constant. The interior of the lattice is defined by the cartesian product 


$$
R(h)=R_{1}(h) \times R_{2}(h)
$$

where

$$
R_{i}(h)=\left\{x_{i} \mid x_{i}=h, 2 h, \cdots, 1-h\right\} .
$$

The boundary of the lattice is defined by

$$
\partial R(h)=\partial R_{1}(h) \times R_{2}(h) \cup R_{1}(h) \times \partial R_{2}(h),
$$

where

$$
\partial R_{i}(h)=\left\{x_{i} \mid x_{i}=0,1\right\} .
$$

Our difference approximation to (1.1) is the standard five point approximation

$$
\begin{aligned}
-D_{-x_{1}}\left(a_{1}\left(x+(h / 2) e_{1}\right) D_{+x_{1}} u(x)\right) & \\
-D_{-x_{2}}\left(a_{2}\left(x+(h / 2) e_{2}\right) D_{+x_{2}} u(x)\right)+c(x) u(x) & =f(x), \quad x \in R(h), \\
u(x) & =g(x), \quad x \in \partial R(h),
\end{aligned}
$$

where $e_{i}$ is the unit vector in the direction of the positive $i$ th coordinate axis and

$$
h D_{ \pm x_{i}} u(x)= \pm\left(u\left(x \pm h e_{i}\right)-u(x)\right) .
$$

Introduce the operators $Q_{i}$ defined by

$$
\begin{aligned}
Q_{i} u(x) & =-D_{-x_{i}}\left(a_{i}\left(x+(h / 2) e_{i}\right) D_{+x_{i}} u(x)\right)+c_{i}(x) u(x), & & x \in R(h) \\
& =0, & & x \in \partial R(h),
\end{aligned}
$$

where $c_{1}(x)$ and $c_{2}(x)$ are real valued sufficiently smooth functions which fulfill $c_{1}(x)+c_{2}(x)=c(x)$. If we consider $u(x)$ to be a vector with a component corresponding to each point of $R(h)$, we see easily that the centered difference structure implies that the operators $Q_{i}, i=1,2$, correspond to symmetric matrices and that they thus only have real eigenvalues. We will always assume that these eigenvalues are bounded from below by a strictly positive constant for all $h \leqq h_{0}$. We will refer to this condition as the eigenvalue condition.

We will discuss the eigenvalues of $Q_{i}, i=1,2$, in Section 3 and give a sufficient condition in terms of the coefficients of $Q_{i}$ to ensure that the eigenvalue condition is fulfilled. In the same section we will also show that (2.1) has a unique solution and that the operators on the left-hand sides of our ADI-scheme

$$
\begin{gathered}
\left(\rho_{n}(x, h)+h^{2} Q_{1}\right) u_{n+1 / 2}=\left(\rho_{n}(x, h)-h^{2} Q_{2}\right) u_{n}+h^{2} f, \\
\left(\rho_{n}(x, h)+h^{2} Q_{2}\right) u_{n+1}=\left(\rho_{n}(x, h)-h^{2} Q_{1}\right) u_{n+1 / 2}+h^{2} f, \\
u_{1}(x)=\cdots=u_{n+1 / 2}(x)=u_{n+1}(x)=g(x), \quad x \in \partial R(h), \\
n=1,2, \cdots, \quad u_{1}(x) \text { given, }
\end{gathered}
$$

have well defined inverses if $\rho_{n}(x, h)>0$. Here $u_{1}(x)$ is the initial approximation to the solution of $(2.1)$ and $\rho_{n}(x, h)$ are the iteration parameters. We will assume that these parameters satisfy

$$
\rho_{1}(x, h)>\rho_{2}(x, h)>\cdots>\rho_{m}(x, h),
$$


and

$$
\rho_{n_{1}}(x, h)=\rho_{n}(x, h) \quad \text { if } \quad n_{1}=n \bmod (m) .
$$

We will also assume that the parameters $\rho_{n}(x, h)$ can be written as a product of a fixed function of $x$ and a function which depends on $h$ and $n$. To simplify the notations later, we let $\rho_{n}(x, h)$ have the form

$$
\rho_{n}(x, h)=b^{2}(x) h^{2}\left(k_{n}(h)\right)^{-1}
$$

where $k_{n}(h)>0$ and $b(x)$ is a sufficiently smooth, strictly positive function.

Now suppose that $u_{1}, u_{2}, \cdots$ converge to a limit. This limit must then be equal to the solution of (2.1). This fact enables us to reduce our problem to the study of the convergence to zero of the solution of the following error equation,

$$
\begin{aligned}
& \left(\rho_{n}(x, h)+h^{2} Q_{1}\right) \epsilon_{n+1 / 2}=\left(\rho_{n}(x, h)-h^{2} Q_{2}\right) \epsilon_{n}, \\
& \left(\rho_{n}(x, h)+h^{2} Q_{2}\right) \epsilon_{n+1}=\left(\rho_{n}(x, h)-h^{2} Q_{1}\right) \epsilon_{n+1 / 2},
\end{aligned} \quad n=1,2, \cdots,
$$

with

$$
\epsilon_{\nu}=u_{\nu}-u,
$$

where $u$ is the solution of (2.1).

In the following we shall work almost exclusively with mesh functions which are zero for $x \in \partial R(h)$. We therefore introduce the space

$$
U_{0}=\{u \mid u(x)=0, x \in \partial R(h)\},
$$

with an inner product and a norm defined by

$$
(u, v)=\sum_{x \in R(h)} h^{2} \bar{u}(x) v(x), \quad\|u\|^{2}=(u, u) .
$$

By $\Omega(A)$ we denote the spectral radius of the operator $A$. It is well known that

$$
\Omega(A) \leqq\|A\| .
$$

For $p \geqq q$ we will write $\prod_{q}^{p} A_{\nu}$ for $A_{p} A_{p-1} \cdots A_{q}$. We also define $\prod_{q}^{q-1} A_{\nu}=I=$ the identity operator.

Consider (2.4). To decide the convergence of our method we want to estimate

$$
\Omega_{m}=\Omega\left\{\prod_{1}^{m}\left(\rho_{\nu}+h^{2} Q_{2}\right)^{-1}\left(\rho_{\nu}-h^{2} Q_{1}\right)\left(\rho_{\nu}+h^{2} Q_{1}\right)^{-1}\left(\rho_{\nu}-h^{2} Q_{\Sigma}\right)\right\} .
$$

Using (2.3) we see that

$$
\begin{aligned}
\rho_{\nu}-h^{2} Q_{i} & =\frac{h^{2}}{k_{\nu}(h)} b(x)\left(1-k_{\nu} Q_{i}^{\prime}\right) b(x) \quad \text { and } \\
\left(\rho_{\nu}+h^{2} Q_{i}\right)^{-1} & =\frac{k_{\nu}(h)}{h^{2}}(b(x))^{-1}\left(1+k_{\nu} Q_{i}^{\prime}\right)^{-1}(b(x))^{-1},
\end{aligned}
$$

where

$$
Q_{i}{ }^{\prime}=(b(x))^{-1} Q_{i}(b(x))^{-1}, \quad i=1,2 .
$$


(2.6) tells us that

$\Omega_{m}=\Omega\left\{(b(x))^{-1}\left(\prod_{1}^{m}\left(1+k_{\nu} Q_{2}{ }^{\prime}\right)^{-1}\left(1-k_{\nu} Q_{1}{ }^{\prime}\right)\left(1+k_{\nu} Q_{1}{ }^{\prime}\right)^{-1}\right.\right.$

$$
\left.\left.\times\left(1-k_{\nu} Q_{2}^{\prime}\right)\right) b(x)\right\}
$$

By a similarity transformation we get

$$
\Omega_{m}=\Omega\left\{\prod_{1}^{m}\left(1+k_{\nu} Q_{2}{ }^{\prime}\right)^{-1}\left(1-k_{\nu} Q_{1}{ }^{\prime}\right)\left(1+k_{\nu} Q_{1}{ }^{\prime}\right)^{-1}\left(1-k_{\nu} Q_{2}{ }^{\prime}\right)\right\} .
$$

The spectral radius $\Omega_{m}$ will eventually be estimated by using (2.5). Before this is done we will however carry out still another similarity transformation. Thus let us introduce

$$
\begin{aligned}
S_{\nu}= & \left(1+k_{m} Q_{2}{ }^{\prime}\right)\left(1+k_{\nu} Q_{2}{ }^{\prime}\right)^{-1}\left(1-k_{\nu} Q_{1}{ }^{\prime}\right)\left(1+k_{\nu} Q_{1}{ }^{\prime}\right)^{-1} \\
& \times\left(1-k_{\nu} Q_{2}{ }^{\prime}\right)\left(1+k_{m}{Q_{2}}^{\prime}\right)^{-1} .
\end{aligned}
$$

It is easily seen that

$$
\Omega_{m}=\Omega\left\{\prod_{1}^{m} S_{\nu}\right\} \leqq\left\|\prod_{1}^{m} S_{\nu}\right\|
$$

We will write $\prod_{1}^{m} S_{\nu}$ as the sum of two terms,

$$
\prod_{1}^{m} S_{\nu}=\prod_{1}^{m} T_{\nu 1} \prod_{1}^{m} T_{\nu 2}+R
$$

where

$$
T_{\nu i}=\left(1+k_{\nu} Q_{i}{ }^{\prime}\right)^{-1}\left(1-k_{\nu} Q_{i}{ }^{\prime}\right)
$$

and the remainder $R$ is defined by (2.9). In commutative cases $R=0$. From $(2.8)$ and (2.9) we obtain,

$$
\Omega_{m} \leqq\left\|\prod_{1}^{m} T_{\nu 1}\right\| \cdot\left\|\prod_{1}^{m} T_{\nu 2}\right\|+\|R\| .
$$

We will show in Section 4 how to choose the $k_{\nu}$ to make the first of these terms small and in Sections 5 and 6 how to choose $b(x)$ to make $\|R\|$ very small. The first term in (2.10) can be treated just as if our problem were commutative.

As will be shown in Section $3, Q_{i}{ }^{\prime}$ as well as $Q_{i}$ are selfadjoint operators and they therefore only have real eigenvalues. The bounds which we can get for $\left\|\prod_{i}^{m} T_{\nu i}\right\|$ depend on lower and upper bounds for these eigenvalues. Suppose that we know that the eigenvalues of $Q_{i}{ }^{\prime}, i=1,2$, lie between $\alpha$ and $\beta$ where $0<\alpha<\beta$. We are then able to state our main result.

TheOREm 1. If $b^{2}(x)=a_{1}(x)$ and we choose $k_{\nu}$ conveniently we have

$$
\Omega_{m} \leqq\left(\frac{1-(\alpha / \beta)^{1 / 2 m}}{1+(\alpha / \beta)^{1 / 2 m}}\right)^{2}+O\left(h^{3 / 2 m}\right) .
$$

We give estimates for $\alpha$ and $\beta$ in Section 3. There we will also show that

$$
\alpha / \beta=O\left(h^{2}\right)
$$


if the eigenvalue condition is fulfilled. From (2.11) and (2.12) follows

$$
\Omega_{m} \leqq\left(\frac{1-q(h)}{1+q(h)}\right)^{2}+O\left(h^{3 / 2 m}\right),
$$

where $0<q(h)=O\left(h^{1 / m}\right)$. Compare (1.2).

The case $a_{1}=a_{2}$ is of course of particular interest in many applications. In that case we can prove a somewhat stronger theorem.

Theorem 2. Suppose that $a_{1}(x)=a_{2}(x)$. Then if $b^{2}(x)=a_{1}(x)$ and $k_{\nu}$ are chosen conveniently we have

$$
\left\|b \epsilon_{m+1}\right\| \leqq\left\{\left(\frac{1-(\alpha / \beta)^{1 / 2 m}}{1+(\alpha / \beta)^{1 / 2 m}}\right)^{2}+O\left(h^{3 / 2 m}\right)\right\}\left\|b \epsilon_{1}\right\| .
$$

Remark. If we consider equations of type (1.1) for which the coefficients can be extended to be periodic (of period 2 in $x_{1}$ and $x_{2}$ ), even, sufficiently smooth functions for all values of $x$ it can be shown that we do not necessarily need to choose $b^{2}(x)=a_{1}(x)$ to achieve rapid convergence. In fact it suffices to make $\max \left|\partial / \partial x_{2}\left(a_{1} / b^{2}\right)\right|$ sufficiently small. The proof of this result is quite involved and we do not give it in this paper. Numerical experiments indicate that an upper bound for $\left|\partial / \partial x_{2}\left(a_{1} / b^{2}\right)\right|$ is needed to guarantee convergence for small values of $m$.

3. The Eigenvalues of $Q_{i}$ and $Q_{i}{ }^{\prime}$. We begin this section by proving

Proposition 3.1. $Q_{i}$ and $Q_{i}{ }^{\prime}, i=1,2$, are selfadjoint operators, i.e.

$$
\left(Q_{i} u, v\right)=\left(u, Q_{i} v\right), \quad\left(Q_{i}{ }^{\prime} u, v\right)=\left(u, Q_{i}{ }^{\prime} v\right),
$$

for all $u, v \in U_{0} . Q_{i}$ and $Q_{i}{ }^{\prime}$ thus have only real eigenvalues.

Proof. That $\left(Q_{i} u, v\right)=\left(u, Q_{i} v\right)$ follows immediately from the definitions of the inner product and $Q_{i}$, the fact that $u, v \in U_{0}$ and a summation by parts. The fact that $\left(Q_{i}{ }^{\prime} u, v\right)=\left(Q_{i} b^{-1} u, b^{-1} v\right)$ and $\left(u, Q_{i}{ }^{\prime} v\right)=\left(b^{-1} u, Q_{i} b^{-1} v\right)=\left(Q_{i} b^{-1} u, b^{-1} v\right)$ proves the second statement of the theorem.

We next give an estimate of the eigenvalues of $Q_{i}$.

Proposition 3.2. Suppose that $\lambda$ is an eigenvalue of $Q_{i}$ for a fixed value of $x_{j} \in R_{j}$, $j \neq i$. Then

$$
\begin{aligned}
& \min _{0 \leqq x_{i} \leqq 1} a_{i}(x) 4 \sin ^{2}(\pi h / 2) / h^{2}+\min _{0 \leqq x_{i} \leqq 1} c_{i}(x) \leqq \lambda \\
& \leqq \max _{0 \leqq x_{i} \leqq 1} a_{i}(x) 4 \cos ^{2}(\pi h / 2) / h^{2}+\max _{0 \leqq x_{i} \leqq 1} c_{i}(x) .
\end{aligned}
$$

Before we give a proof we state a corollary to Proposition 3.2.

Corollary. The eigenvalue condition is fulfilled if

$$
c_{i}(x)>-\min a_{i}(x) 4 \sin ^{2}\left(\pi h_{0} / 2\right) / h_{0}{ }^{2}, \quad i=1,2 .
$$

In this inequality the min are taken for all values of $x$ in the closed unit square.

Proof of the proposition. We will use the following notation

$$
\begin{aligned}
(u, v)_{i: r, s} & =\sum_{r h \leqq x_{i} \leqq s h} h \bar{u}(x) v(x), \\
\|u\|_{i: r, s}^{2} & =(u, u)_{i: r, s}
\end{aligned}
$$


where $0 \leqq r<s \leqq N$ and $u$ and $v$ are mesh functions defined on $R_{i}(h) \cup \partial R_{i}(h)$. Let $\phi\left(x_{i}\right) \in U_{0}$ be an eigenfunction of $Q_{i}$ for which $\|\phi\|_{i: 1, N-1}=1$ and

$$
Q_{i} \phi=\lambda \phi, \quad x_{j} \in R_{j} \text { fixed, } j \neq i .
$$

Then

$\lambda=(\lambda \phi, \phi)_{i: 1, N-1}=\left(-D_{-x_{i}} a_{i}\left(x+(h / 2) e_{i}\right) D_{+x_{i}} \phi, \phi\right)_{i: 1, N-1}+\left(c_{i} \phi, \phi\right)_{i: 1, N-1}$.

Writing out the sums it is easy to see that

$\left(-D_{-x_{i}} a_{i}\left(x+(h / 2) e_{i}\right) D_{+x_{i}} \phi, \phi\right)_{i: 1, N-1}=\left(a_{i}\left(x+(h / 2) e_{i}\right) D_{+x_{i}} \phi, D_{+x_{i}} \phi\right)_{i: 0, N-1}$.

Thus

$\min _{0 \leqq x_{i} \leqq 1} a_{i} \mid D_{+x_{i}} \phi\left\|_{i: 0, N-1}^{2}+\min _{0 \leqq x_{i} \leqq 1} c_{i} \leqq \lambda \leqq \max _{0 \leqq x_{i} \leqq 1} a_{i}\right\| D_{+x_{i}} \phi \|_{i: 0, N-1}^{2}+\max _{0 \leqq x_{i} \leqq 1} c_{i}$.

To finish our proof, we show that

$$
4 \sin ^{2}(\pi h / 2) / h^{2} \leqq\left\|D_{+x_{i}} \phi\right\|_{i: 0, N-1}^{2} \leqq 4 \cos ^{2}(\pi h / 2) / h^{2} .
$$

To do this we develop $\phi\left(x_{i}\right)$ in a discrete Fourier series

$$
\phi\left(x_{i}\right)=\sum_{\nu=1}^{N-1} \alpha_{\nu} \sin \pi x_{i} \nu
$$

It is easy to show that $\left(\sin \pi x_{i} \nu, \sin \pi x_{i} \mu\right)_{i: 1, N-1}=\frac{1}{2} \delta_{\nu \mu}$ if $1 \leqq \nu, \mu \leqq N-1$. We know that

$$
\|!\|_{i: 1, N-1}^{2}=\frac{1}{2} \sum_{\nu=1}^{N-1}\left|\alpha_{\nu}\right|^{2}=1
$$

Furthermore

$$
\begin{aligned}
\left\|D_{+x_{i}} \phi\right\|_{i: 0, N-1}^{2} & =-\left(\phi, D_{-x_{i}} D_{+x_{i}} \phi\right)_{i: 1, N-1} \\
& =\frac{1}{2} \sum_{\nu=1}^{N-1} 4 \sin ^{2}(\pi \nu h / 2) / h^{2}\left|\alpha_{\nu}\right|^{2},
\end{aligned}
$$

which proves (3.1).

The eigenvalues of $Q_{i}{ }^{\prime}$ ('an be estimated in terms of the eigenvalues of $Q_{i}$.

Proposition 3.3. Suppose that for a given $x_{j}, j \neq i$, the eigenvalues of $Q_{i}$ fulfill

$$
0<\alpha\left(x_{j}\right) \leqq \lambda \leqq \beta\left(x_{j}\right) .
$$

Then the eigenvalues $\lambda^{\prime}$ of $Q_{i}{ }^{\prime}$ satisfy

$$
0<\alpha\left(x_{j}\right) / \max _{0 \leqq x_{i} \leqq 1} b^{2}(x) \leqq \lambda^{\prime} \leqq \beta\left(x_{j}\right) / \min _{0 \leqq x_{i} \leqq 1} b^{2}(x) .
$$

Proof. Let

$$
Q_{i}{ }^{\prime} \phi=\lambda^{\prime} \phi
$$

where $\phi \|_{i: 1, N-1}=1$. Since $Q_{i}$ is a selfadjoint operator $\alpha\left(x_{j}\right)\|\Psi\|_{i: 1, N-1}^{2} \leqq$ $\left(Q_{i} \Psi, \Psi\right)_{i: 1, N-1} \leqq \beta\left(x_{j}\right)\|\Psi\|_{i: 1, N-1}^{2}$, for all $\Psi \in U_{0}$. By the definition of $Q_{i}^{\prime}$ we get

$$
\begin{aligned}
\lambda^{\prime}=\left(\lambda^{\prime} \phi, \phi\right)_{i: 1, N-1} & =\left(Q_{i}{ }^{\prime} \phi, \phi\right)_{i: 1, N-1} \\
& =\left(Q_{i}(b)^{-1} \phi,(b)^{-1} \phi\right)_{i: 1, N-1} .
\end{aligned}
$$


Thus

$$
\begin{aligned}
\left(\alpha\left(x_{j}\right) / \max _{0 \leqq x_{i} \leqq 1} b^{2}(x)\right) & \leqq \alpha\left(x_{j}\right)\left\|b^{-1} \phi\right\|_{i: 1, N-1}^{2} \\
& \leqq\left(Q_{i} b^{-1} \phi, b^{-1} \phi\right)_{i: 1, N-1} \leqq \beta\left(x_{j}\right)\left\|b^{-1} \phi\right\|_{i: 1, N-1}^{2} \\
& \leqq\left(\beta\left(x_{j}\right) / \min _{0 \leqq x_{i} \leqq 1} b^{2}(x)\right)
\end{aligned}
$$

which completes the proof.

We may use Propositions 3.2 and 3.3 to estimate the quantities $\alpha$ and $\beta$ which were introduced in Section 2 . These propositions also tell us that if the eigenvalue condition is fulfilled we always have $\alpha / \beta=O\left(h^{2}\right)$. It should however be pointed out that the values of $\alpha$ and $\beta$ which can be computed from Propositions 3.2 and 3.3 often are rather pessimistic and that it is sometimes advisable to try to get better bounds by some other method since the error estimates are then improved. It is also known from experience that the efficiency of the ADI-scheme depends very much on the choice of $\alpha$ and $\beta$.

We next show some simple consequences of the eigenvalue condition.

Proposition 3.4. Equation (2.1) has a unique solution.

Proof. (2.1) corresponds to a system of linear equations with as many equations as unknowns. We therefore only have to show that $u \equiv 0$ is the only solution to (2.1) for $f(x)=g(x) \equiv 0$. Suppose that $v(x) \not \equiv 0$ and

$$
\left(Q_{1}+Q_{2}\right) v=0 \text {. }
$$

This is not possible, since the $Q_{i}$ are selfadjoint operators with strictly positive eigenvalues. That is, there is a $c>0$ such that

$$
\left(\left(Q_{1}+Q_{2}\right) v, v\right) \geqq c\|v\|^{2},
$$

for all $v \in U_{0}$.

This contradiction establishes the proposition.

Proposition 3.5.

$$
\begin{aligned}
\left(\rho_{n}(x, h)+h^{2} Q_{i}\right) u\left(x_{i}\right) & =f\left(x_{i}\right), & x_{i} \in R_{i}(h), \\
u(0)=u_{0}, \quad u(1) & =u_{1} &
\end{aligned}
$$

has a unique solution.

Proof. Observe that by (2.6)

$$
\rho_{n}(x, h)+h^{2} Q_{i}=\frac{h^{2}}{k_{n}(h)} b(x)\left(1+k_{n} Q_{i}^{\prime}\right) b(x) .
$$

By Proposition 3.3 the eigenvalues of $Q_{i}{ }^{\prime}$ are positive, hence this operator has a unique inverse.

Proposition 3.5 shows that $u_{3 / 2}, u_{2}, \cdots$, defined by (2.2) are all well defined.

The following lemmas will be used later.

Lemma 3.1. For any $k>0$

$$
\begin{aligned}
\left\|\left(1+k Q_{i}{ }^{\prime}\right)^{-1}\right\| & \leqq \frac{1}{1+k \alpha}<1, \\
\left\|\left(1+k Q_{i}^{\prime}\right)^{-1} k Q_{i}{ }^{\prime}\right\| & \leqq \frac{k \beta}{1+k \beta}<1, \\
\left\|\left(1+k Q_{i}{ }^{\prime}\right)^{-1}\left(1-k Q_{i}^{\prime}\right)\right\| & \leqq \max \left(\frac{1-k \alpha}{1+k \alpha}, \frac{k \beta-1}{k \beta+1}\right)<1 .
\end{aligned}
$$


Proof. $Q_{i}{ }^{\prime}$ are selfadjoint operators and they therefore have complete sets of orthonormal eigenfunctions. Develop $u \in U_{0}$ in series of eigenfunctions of $Q_{i}^{\prime}$.

$$
u=\sum_{\nu=1}^{N-1} \alpha_{\nu}\left(x_{j}\right) \phi_{\nu}\left(x_{i}\right), \quad j \neq i, \quad\left(\phi_{\nu}, \phi_{\mu}\right)_{i: 1, N-1}=\delta_{\nu \mu} .
$$

Now

$$
\left(1+k Q_{i}^{\prime}\right)^{-1} u=\sum_{\nu=1}^{N-1} \frac{\alpha_{\nu}\left(x_{j}\right)}{1+k \lambda_{\nu}\left(x_{j}\right)} \cdot \phi_{\nu}\left(x_{i}\right)
$$

where $\lambda_{\nu}\left(x_{j}\right)$ is the eigenvalue corresponding to $\phi_{\nu}\left(x_{i}\right)$. Thus

$$
\begin{aligned}
\left\|\left(1+k Q_{i}{ }^{\prime}\right)^{-1} u\right\|^{2} & =\sum_{\nu=1}^{N-1}\left\|\alpha_{\nu} /\left(1+k \lambda_{\nu}\right)\right\|_{j: 1, N-1}^{2} \\
& \leqq\left(\frac{1}{1+k \alpha}\right)^{2} \sum_{\nu=1}^{N-1}\left\|\alpha_{\nu}\right\|_{j: 1, N-1}^{2}=\left(\frac{1}{1+k \alpha}\right)^{2}\|u\|^{2} .
\end{aligned}
$$

The other inequalities follow in the same way.

Lemma 3.2 .

$$
\left\|\prod_{1}^{m} T_{\nu i}\right\| \leqq \max _{\alpha \leqq \lambda \leqq \beta} \prod_{1}^{m}\left|\frac{1-k_{\nu} \lambda}{1+k_{\nu} \lambda}\right| .
$$

Proof. Cf. the proof of Lemma 3.1 .

4. The Choice of $k_{v}$. It is obvious from Lemma 3.2 why we are interested in making

$$
\max _{\alpha \leqq \lambda \leqq \beta} \prod_{1}^{m}\left|\frac{1-k_{\nu} \lambda}{1+k_{\nu} \lambda}\right|
$$

small. This problem has been extensively treated in the literature and hence we will give it only a brief treatment. We refer the reader to [2], [5] or [6] for details.

The optimal as well as several other good choices of $k_{\nu}$ are known. The perhaps simplest choice is

$$
k_{\nu}=\frac{1}{\beta}\left(\frac{\beta}{\alpha}\right)^{(2 \nu-1) / 2 m}, \quad \nu=1,2, \cdots, m \text {, }
$$

which corresponds to the parameters suggested in the original paper by Peaceman and Rachford [3].

With this choice we get

$$
\begin{aligned}
\left\|\prod_{1}^{m} T_{\nu i}\right\| & \leqq \max _{\alpha \leqq \lambda \leqq \beta} \prod_{1}^{m}\left|\frac{1-k_{\nu} \lambda}{1+k_{\nu} \lambda}\right| \leqq \frac{1-(\alpha / \beta)^{1 / 2 m}}{1+(\alpha / \beta)^{1 / 2 m}} \\
& =\frac{1-q(h)}{1+q(h)}, \text { where } 0<q(h)=O\left(h^{1 / m}\right) .
\end{aligned}
$$

The last equality follows from the fact that $\alpha / \beta=O\left(h^{2}\right)$. Using the results of Section 3 we see that

$$
k_{\nu}=O\left(h^{(2(m-\nu)+1) / m}\right), \quad \quad \nu=1,2, \cdots m .
$$


It is easy to show that (4.1) and (4.2) are true also for the optimal parameters due to Jordan. Cf. Wachspress [6]. In the proof of our theorems we will suppose that the $k_{\nu}$ are chosen so that (4.1) and (4.2) hold. But we could by our technique prove convergence when $h \rightarrow 0$ for all sets of $k_{\nu}$ for which

$$
\max _{\alpha \leqq \lambda \leqq \beta} \prod_{1}^{m}\left|\frac{1-k_{\nu} \lambda}{1+k_{\nu} \lambda}\right|^{2} \leqq\left(\frac{1-c(\alpha, \beta)}{1+c(\alpha, \beta)}\right)^{2}
$$

where $0<c(\alpha, \beta)<1$,

$$
h\left(k_{m}\right)^{1 / 2} / c(\alpha, \beta) \rightarrow 0 \quad \text { when } h \rightarrow 0,
$$

and for $\nu=1,2, \cdots, m-1$

$$
\left(k_{\nu}\right)^{1 / 2} / c(\alpha, \beta) \rightarrow 0 \quad \text { when } h \rightarrow 0 .
$$

5. A Fundamental Inequality. In this section we will derive an inequality for the operators

$$
K_{\nu \mu}=k_{\nu} k_{\mu}\left(1+k_{\nu} Q_{1}{ }^{\prime}\right)^{-1}\left(Q_{1}{ }^{\prime} Q_{2}{ }^{\prime}-Q_{2}{ }^{\prime} Q_{1}{ }^{\prime}\right)\left(1+k_{\mu} Q_{2}{ }^{\prime}\right)^{-1}
$$

which will play an important part in the proofs of our theorems.

Lemma 5.1.

$$
\begin{aligned}
\left\|K_{\nu \mu}\right\| \leqq 2\left(k_{\mu}\right)^{1 / 2} & \max _{0 \leqq x_{1}, x_{2} \leqq 1}\left|b(x) \frac{a_{2}(x)}{a_{1}(x)} \frac{\partial}{\partial x_{2}}\left(a_{1}(x) / b^{2}(x)\right)\right| \\
& \times\left(4 \min _{0 \leqq x_{1}, x_{2} \leqq 1} a_{2}\left(1+k_{\mu} \min _{0 \leqq x_{1}, x_{2} \leqq 1}\left(b^{-2} c_{2}\right)\right)\right)^{1 / 2} \\
+ & 2\left(k_{\nu}\right)^{1 / 2} \max _{0 \leqq x_{1}, x_{2} \leqq 1}\left|b(x) \frac{a_{1}(x)}{a_{2}(x)} \frac{\partial}{\partial x_{1}}\left(a_{2}(x) / b^{2}(x)\right)\right| \\
& \times\left(4 \min _{0 \leqq x_{1}, x_{2} \leqq 1} a_{1}\left(1+k_{\nu} \min _{0 \leqq x_{1}, x_{2} \leqq 1}\left(b^{-2} c_{1}\right)\right)\right)^{-1 / 2} \\
+ & k_{\mu} \max _{0 \leqq x_{1}, x_{2} \leqq 1}\left|\frac{1}{a_{1}(x)} \cdot \frac{\partial}{\partial x_{2}}\left(a_{2}(x) \frac{\partial}{\partial x_{2}}\left(a_{1}(x) / b^{2}(x)\right)\right)\right| \\
+ & k_{\nu} \max _{0 \leqq x_{1}, x_{2} \leqq 1}\left|\frac{1}{a_{2}(x)} \cdot \frac{\partial}{\partial x_{1}}\left(a_{1}(x) \frac{\partial}{\partial x_{1}}\left(a_{2}(x) / b^{2}(x)\right)\right)\right| \\
+ & O\left(h\left(k_{\nu}\right)^{1 / 2}+h\left(k_{\mu}\right)^{1 / 2}+\left(k_{\nu}\right)^{1 / 2} k_{\mu}+\left(k_{\mu}\right)^{1 / 2} k_{\nu}\right) .
\end{aligned}
$$

The following corollary follows immediately from the lemma.

Corollary. If $b^{2}=a_{1}$ we have

$$
\left\|K_{\nu \mu}\right\|=O\left(\left(k_{\nu}\right)^{1 / 2}+h\left(k_{\mu}\right)^{1 / 2} .\right.
$$

If furthermore $a_{1}=a_{2}$ we have

$$
\left\|K_{\nu \mu}\right\|=O\left(h\left(k_{\nu}\right)^{1 / 2}+h\left(k_{\mu}\right)^{1 / 2}+k_{\nu}\left(k_{\mu}\right)^{1 / 2}+k_{\mu}\left(k_{\nu}\right)^{1 / 2}\right) .
$$

Proof of the lemma. We first consider the operator $Q_{1}{ }^{\prime} Q_{2}{ }^{\prime}-Q_{2}{ }^{\prime} Q_{1}{ }^{\prime}$ which maps $U_{0}$ into $U_{0}$. For $x \in R(h)$ this operator is defined as the commutator of two second order difference operators and it can therefore be expressed as a third order differ- 
ence operator. Using discrete counterparts of Leibnitz's rule we may write

$$
\begin{aligned}
\left(Q_{1}{ }^{\prime} Q_{2}{ }^{\prime}-\right. & \left.Q_{2}{ }^{\prime} Q_{1}{ }^{\prime}\right) u= \\
& Q_{1}{ }^{\prime}\left(A_{21}^{+}(x, h) D_{+x_{2}} b^{-1}+A_{21}^{-}(x, h) D_{-x_{2}} b^{-1}+A_{20}(x, h)\right) u \\
& +\left(b^{-1} D_{+x_{1}} A_{12}^{+}(x, h)+b^{-1} D_{-x_{1}} A_{12}^{-}(x, h)+A_{02}(x, h)\right) Q_{2}^{\prime} u \\
& +b^{-1} D_{+x_{1}} A_{11}^{++}(x, h) D_{+x_{2}} b^{-1} u+b^{-1} D_{-x_{1}} A_{11}^{-+}(x, h) D_{+x_{2}} b^{-1} u \\
& +b^{-1} D_{+x_{1}} A_{11}^{+-}(x, h) D_{-x_{2}} b^{-1} u+b^{-1} D_{-x_{1}} A_{11}^{-}(x, h) D_{-x_{2}} b^{-1} u \\
& +b^{-1} D_{+x_{1}} A_{10}^{+}(x, h) u+b^{-1} D_{-x_{1}} A_{10}^{-}(x, h) u+A_{01}^{+}(x, h) D_{+x_{2}} b^{-1} u \\
& +A_{01}^{-}(x, h) D_{-x_{2}} b^{-1} u+A_{00}(x, h) u,
\end{aligned}
$$

for $x \in R(h)$ and $u \in U_{0}$. Formulas for the coefficients $A_{21}^{+}(x, h)$ etc. can be given in terms of $a_{1}, a_{2}, b, c_{1}$ and $c_{2}$ and certain of their divided differences. Because of our assumptions on the smoothness of $a_{1}$, etc. we see that all the coefficients in (5.1) are uniformly bounded functions of $x$ and $h$. It can be shown that

$$
\begin{aligned}
A_{21}^{+}(x, h) & =-b(x) \frac{a_{2}(x)}{a_{1}(x)} \cdot \frac{\partial}{\partial x_{2}}\left(a_{1}(x) / b^{2}(x)\right)+O(h), \\
A_{21}^{-}(x, h) & =A_{21}^{+}(x, h)+O(h), \\
A_{12}^{+}(x, h) & =b(x) \frac{a_{1}(x)}{a_{2}(x)} \frac{\partial}{\partial x_{1}}\left(a_{2}(x) / b^{2}(x)\right)+O(h), \\
A_{12}^{-}(x, h) & =A_{12}^{+}(x, h)+O(h), \\
A_{20}(x, h) & =-\frac{1}{a_{1}(x)} \frac{\partial}{\partial x_{2}}\left(a_{2}(x) \frac{\partial}{\partial x_{2}}\left(a_{1}(x) / b^{2}(x)\right)\right)+O(h), \\
A_{02}(x, h) & =-\frac{1}{a_{2}(x)} \frac{\partial}{\partial x_{1}}\left(a_{1}(x) \frac{\partial}{\partial x_{1}}\left(a_{2}(x) / b^{2}(x)\right)\right)+O(h) .
\end{aligned}
$$

We can extend the formula (5.1) to be valid even for $x \in \partial R(h)$ if we replace $D_{ \pm x_{i}}$ by $\tilde{D}_{ \pm x_{i}}$ in (5.1), where

$$
\begin{aligned}
\tilde{D}_{ \pm x_{i}} u & =D_{ \pm x_{i}} u, & x \in R(h), \\
& =0, & x \in \partial R(h) .
\end{aligned}
$$

The $\tilde{D}_{ \pm x_{i}}$ are thus mappings of $U_{0}$ into $U_{0}$. We extend our coefficients, when needed, by defining them to be zero for $x \in \partial R(h)$. Our formula (5.1) will then not only give a correct representation for $\left(Q_{1}{ }^{\prime} Q_{2}{ }^{\prime}-Q_{2}{ }^{\prime} Q_{1}{ }^{\prime}\right) u$ in the interior of our mesh but will also give a representation of $Q_{1}{ }^{\prime}{ }_{2}{ }^{\prime}-Q_{2}{ }^{\prime} Q_{1}{ }^{\prime}$ as the sum of operators which have the form

$$
T_{1} A(x, h) T_{2}
$$

where the $T_{i}, i=1,2$, map $U_{0}$ into $U_{0}, T_{i}$ only operates in the $x_{i}$-direction and $A(x, h)$ is a uniformly bounded function. Now

$$
\begin{aligned}
& \left\|k_{\nu} k_{\mu}\left(1+k_{\nu} Q_{1}{ }^{\prime}\right)^{-1} T_{1} A(x, h) T_{2}\left(1+k_{\mu} Q_{2}{ }^{\prime}\right)^{-1}\right\| \\
& \quad \leqq \max _{0 \leqq x_{1}, x_{2} \leqq 1 ; h \leqq h_{0}}|A(x, h)| \cdot\left\|\left(1+k_{\nu} Q_{1}{ }^{\prime}\right)^{-1} k_{\nu} T_{1}\right\| \cdot\left\|k_{\mu} T_{2}\left(1+k_{\mu} Q_{2}{ }^{\prime}\right)^{-1}\right\| .
\end{aligned}
$$


To finish the proof of Lemma 5.1 we only have to use Lemma 3.1 and the following lemma on the different terms of $K_{\nu \mu}$.

LEMMA 5.2. For $k>0$

$$
\begin{aligned}
\left\|\left(1+k Q_{i}{ }^{\prime}\right)^{-1} k b^{-1} \tilde{D}_{ \pm x_{i}}\right\| & =\left\|k \tilde{D}_{\mp x_{i}} b^{-1}\left(1+k Q_{i}{ }^{\prime}\right)^{-1}\right\| \\
& \leqq k^{1 / 2}\left(4 \min _{0 \leqq x_{1}, x_{2} \leqq 1} a_{i} \cdot\left(1+k \min _{0 \leqq x_{1}, x_{2} \leqq 1} b^{-2} c_{i}\right)\right)^{-1 / 2} .
\end{aligned}
$$

Proof of Lemma 5.2. It is easy to see that $-\tilde{D}_{-x_{i}}$ is the adjoint operator of $\tilde{D}_{+x_{i}}$. Using Proposition 3.1 and the fact that adjoint operators have the same norm we are able to prove the equality in the lemma. Next define

$$
v \equiv\left(1+k Q_{i}{ }^{\prime}\right)^{-1} k^{1 / 2} b^{-1} \tilde{D}_{-x_{i}} u, \quad u \in U_{0}
$$

so that

$$
\left(1+k Q_{i}{ }^{\prime}\right) v=k^{1 / 2} b^{-1} \tilde{D}_{-x_{i}} u .
$$

Form the scalar products of both sides with $v$

$$
\left(v,\left(1+k Q_{i}{ }^{\prime}\right) v\right)_{i: 1, N-1}=\left(v, k^{1 / 2} b^{-1} \tilde{D}_{-x_{i}} u\right)_{i: 1, N-1} .
$$

Observe that $v \in U_{0}$. Hence from considerations very similar to those in Section 3

$$
\begin{aligned}
\left(v,\left(1+k Q_{i}{ }^{\prime}\right) v\right)_{i: 1, N-1} & =\|v\|_{i: 1, N-1}^{2} \\
& -k\left(v, b^{-1} D_{-x_{i}} a_{i}\left(x+(h / 2) e_{i}\right) D_{+x_{i}} b^{-1} v\right)_{i: 1, N-1} \\
& +k\left(v, b^{-2} c_{i} v\right)_{i: 1, N-1} \\
& =\|v\|_{i: 1, N-1}^{2}+k\left(D_{+x_{i}} b^{-1} v, a_{i}\left(x+(h / 2) e_{i}\right) D_{+x_{i}} b^{-1} v\right)_{i: 0, N-1} \\
& +k\left(v, b^{-2} c_{i} v\right)_{i: 1, N-1} \\
& \geqq\left(1+k \min _{0 \leqq x_{1}, x_{2} \leqq 1}\left(b^{-2} c_{i}\right)\right)\|v\|_{i: 1, N-1}^{2} \\
& +\min _{0 \leqq x_{1}, x_{2} \leqq 1} a_{i} k\left\|D_{+x_{i}} b^{-1} v\right\|_{i: 0, N-1}^{2} .
\end{aligned}
$$

Next we show that

$$
\begin{aligned}
\left(v, k^{1 / 2} b^{-1} \tilde{D}_{-x_{i}} u\right)_{i: 1, N-1} & =\left(v, k^{1 / 2} b^{-1} D_{-x_{i}} u\right)_{i: 1, N}=-k^{1 / 2}\left(D_{+x_{i}} b^{-1} v, u\right)_{i: 0, N-1} \\
& \leqq k^{1 / 2}\left\|D_{+x_{i}} b^{-1} v\right\|_{i: 0, N-1}\|u\|_{i: 0, N-1} \\
& \leqq k(C / 2)\left\|D_{+x_{i}} b^{-1} v\right\|_{i: 0, N-1}^{2}+(1 / 2 C)\|u\|_{i: 1, N-1}^{2}
\end{aligned}
$$

for all $C>0$. If we choose $C=2 \min _{0 \leqq x_{1}, x_{2} \leqq 1} a_{i}$ we get

$$
\begin{aligned}
\left\|\left(1+k Q_{i}{ }^{\prime}\right)^{-1} k^{1 / 2} b^{-1} \tilde{D}_{-x_{i}} u\right\|_{i: 1, N-1}^{2} & \\
& \leqq\left\{4 \min _{0 \leqq x_{1}, x_{2} \leqq 1} a_{i} \cdot\left[1+k \min _{0 \leqq x_{1}, x_{2} \leqq 1}\left(b^{-2} c_{i}\right)\right]\right\}^{-1}\|u\|_{i: 1, N-1}^{2} .
\end{aligned}
$$

If we sum this inequality over $R_{j}, j \neq i$, we get one of the inequalities in (5.2). The case with $\tilde{D}_{+x_{i}}$ instead of $\tilde{D}_{-x_{i}}$ can be treated in a quite similar way.

6. Proofs of the Theorems. We will now estimate the norm of the remainder $R$ which was defined by $(2.9)$. 
LEMMA 6.1.

$$
\|R\| \leqq \prod_{\nu=1}^{m-1}\left(1+\left\|R_{\nu}\right\|\right)-1+\sum_{\nu=1}^{m-1}\left\|R_{\nu}{ }^{\prime}\right\|
$$

where

$$
\left\|R_{\nu}\right\| \leqq 2\left\{\left\|K_{\nu \nu}\right\| /\left(1-\left\|K_{\nu \nu}\right\|\right)+\left\|K_{\nu m}\right\| /\left(1-\left\|K_{\nu m}\right\|\right)\right\}
$$

and

$$
\left\|R_{\nu}{ }^{\prime}\right\| \leqq 4 \sum_{\gamma=1}^{\nu}\left\|K_{\gamma, \nu+1}\right\| /\left(1-\left\|K_{\gamma, \nu+1}\right\|\right) .
$$

Combining this lemma with (4.1) and Lemma 5.1 it is easy to finish the proof of Theorem 1 .

Proof of the lemma. The remainder $R$ was defined by

$$
R=\prod_{1}^{m} S_{\nu}-\prod_{1}^{m} T_{\nu 1} \prod_{1}^{m} T_{\nu 2}
$$

where

$S_{\nu}=\left(1+k_{m} Q_{2}{ }^{\prime}\right)\left(1+k_{\nu} Q_{2}{ }^{\prime}\right)^{-1}\left(1-k_{\nu} Q_{1}{ }^{\prime}\right)\left(1+k_{\nu} Q_{1}^{\prime}\right)^{-1}\left(1-k_{\nu} Q_{2}{ }^{\prime}\right)\left(1+k_{m} Q_{2}{ }^{\prime}\right)^{-1}$ and

$$
T_{\nu i}=\left(1+k_{\nu} Q_{i}{ }^{\prime}\right)^{-1}\left(1-k_{\nu} Q_{i}{ }^{\prime}\right)
$$

$R$ can be written as

$$
R=\left(\prod_{1}^{m} S_{\nu}-\prod_{1}^{m} T_{\nu 1} T_{\nu 2}\right)+\left(\prod_{1}^{m} T_{\nu 1} T_{\nu 2}-\prod_{1}^{m} T_{\nu 1} \prod_{1}^{m} T_{\nu 2}\right)
$$

Introduce

$$
R_{\nu} \equiv S_{\nu}-T_{\nu 1} T_{\nu 2} .
$$

Observe that $R_{m}=0$. Obviously for $m=2$

$$
\prod_{1}^{2} S_{\nu}-\prod_{1}^{2} T_{\nu 1} T_{\nu 2}=T_{21} T_{22} R_{1}
$$

whence by Lemma 3.1

$$
\left\|\prod_{1}^{2} S_{\nu}-\prod_{1}^{2} T_{\nu 1} T_{\nu 2}\right\| \leqq\left\|R_{1}\right\|
$$

For $m=3$,

$$
\prod_{1}^{3} S_{\nu}-\prod_{1}^{3} T_{\nu 1} T_{\nu 2}=T_{31} T_{32}\left(T_{21} T_{22} R_{1}+R_{2} T_{11} T_{12}+R_{2} \cdot R_{1}\right)
$$

whence

$$
\begin{aligned}
\left\|\prod_{1}^{3} S_{\nu}-\prod_{1}^{3} T_{\nu 1} T_{\nu 2}\right\| & \leqq\left\|R_{1}\right\|+\left\|R_{2}\right\|+\left\|R_{1}\right\| \cdot\left\|R_{2}\right\| \\
& =\prod_{1}^{2}\left(1+\left\|R_{\nu}\right\|\right)-1
\end{aligned}
$$


In exactly the same way we can show that

$$
\left\|\prod_{1}^{m} S_{\nu}-\prod_{1}^{m} T_{\nu 1} T_{\nu 2}\right\| \leqq \prod_{1}^{m-1}\left(1+R_{\nu} \|\right)-1 .
$$

Next consider the second term in (6.4). We easily get

$$
\prod_{1}^{2} T_{\nu 1} T_{\nu 2}-\prod_{1}^{2} T_{\nu 1} \prod_{1}^{2} T_{\nu 2}=T_{21}\left(T_{22} T_{11}-T_{11} T_{22}\right) T_{12}
$$

In the same way

$$
\begin{aligned}
& \prod_{1}^{3} T_{\nu 1} T_{\nu 2}-\prod_{1}^{3} T_{\nu 1} \prod_{1}^{3} T_{\nu 2} \\
& \quad=T_{31} T_{32}\left(\prod_{1}^{2} T_{\nu 1} T_{\nu 2}-\prod_{1}^{2} T_{\nu 1} \prod_{1}^{2} T_{\nu 2}\right)+T_{31}\left(T_{32} \prod_{1}^{2} T_{\nu 1}-\prod_{1}^{2} T_{\nu 1} T_{32}\right) \prod_{1}^{2} T_{\nu 2} .
\end{aligned}
$$

By (6.6)

$$
\begin{aligned}
\prod_{1}^{3} T_{\nu 1} T_{\nu 2}- & \prod_{1}^{3} T_{\nu 1} \prod_{1}^{3} T_{\nu 2}=T_{31} T_{32} T_{21}\left(T_{22} T_{11}-T_{11} T_{22}\right) T_{12} \\
& +T_{31}\left(T_{32} T_{21}-T_{21} T_{32}\right) T_{11} \prod_{1}^{2} T_{\nu 2}+T_{31} T_{21}\left(T_{32} T_{11}-T_{11} T_{32}\right) \prod_{1}^{2} T_{\nu 2} .
\end{aligned}
$$

Proceeding in this fashion we can show that

$$
\prod_{1}^{m} T_{\nu 1} T_{\nu 2}-\prod_{1}^{m} T_{\nu 1} \prod_{1}^{m} T_{\nu 2}=\sum_{N=1}^{m-1} R_{\mu}^{\prime}
$$

where

$$
R_{\mu}{ }^{\prime} \equiv \prod_{\nu=\mu+2}^{m} T_{\nu 1} T_{\nu 2} \cdot T_{\mu+1,1} \sum_{\gamma=1}^{\mu}\left\{\prod_{\delta=\gamma+1}^{\mu} T_{\delta 1}\left(T_{\mu+1,2} T_{\gamma 1}-T_{\gamma 1} T_{\mu+1,2}\right) \prod_{\rho=1}^{\gamma-1} T_{\rho 1}\right\} \prod_{\nu=1}^{\mu} T_{\nu 2} .
$$

To prove (6.1), we use (6.4), (6.5) and (6.7).

We now prove (6.2). It can be shown by elementary calculations that

$$
\begin{aligned}
R_{\nu}= & -2\left(1-\left(k_{\nu} / k_{m}\right)\right) \\
\times & \left\{\left(1+k_{\nu} Q_{1}^{\prime}\right)^{-1}\left(1+k_{\nu}{Q_{2}}^{\prime}\right)^{-1}-\left(1+k_{\nu} Q_{2}{ }^{\prime}\right)^{-1}\left(1+k_{\nu} Q_{1}{ }^{\prime}\right)^{-1}\right\} \\
& +2\left(k_{m} / k_{\nu}\right)\left(1-\left(k_{\nu} / k_{m}\right)^{2}\right)\left\{\left(1+k_{\nu} Q_{1}{ }^{\prime}\right)^{-1}\left(1+k_{\nu} Q_{2}{ }^{\prime}\right)^{-1}\right. \\
& \left.\quad-\left(1+k_{\nu} Q_{2}{ }^{\prime}\right)^{-1}\left(1+k_{\nu} Q_{1}{ }^{\prime}\right)^{-1}\right\}\left(1+k_{m} Q_{2}{ }^{\prime}\right)^{-1} .
\end{aligned}
$$

We now show how to estimate the norm of the first term in terms of the norm of $K_{\nu \nu}$. Define $P_{\nu}$ and $P_{\nu \nu}$ by

$$
\begin{aligned}
& P_{\nu} \equiv\left(1+k_{\nu} Q_{1}{ }^{\prime}\right)^{-1}(1+\left.k_{\nu} Q_{2}{ }^{\prime}\right)^{-1}-\left(1+k_{\nu} Q_{2}{ }^{\prime}\right)^{-1}\left(1+k_{\nu} Q_{1}{ }^{\prime}\right)^{-1} \\
&=\left(1-k_{\nu} Q_{2}{ }^{\prime}\right)^{-1}\left\{\left(1+k_{\nu} Q_{1}{ }^{\prime}\right)^{-1} k_{\nu}{ }^{2}\left(Q_{1}{ }^{\prime} Q_{2}{ }^{\prime}-{Q_{2}}^{\prime} Q_{1}{ }^{\prime}\right)\right. \\
&\left.\quad \cdot\left(1+k_{\nu} Q_{1}{ }^{\prime}\right)^{-1}\left(1+k_{\nu} Q_{2}{ }^{\prime}\right)^{-1}\right\} \equiv\left(1+k_{\nu} Q_{2}{ }^{\prime}\right)^{-1} P_{\nu \nu} .
\end{aligned}
$$


But

$$
\begin{aligned}
P_{\nu \nu}= & \left(1+k_{\nu} Q_{1}{ }^{\prime}\right)^{-1} k_{\nu}{ }^{2}\left(Q_{1}{ }^{\prime} Q_{2}{ }^{\prime}-Q_{2}{ }^{\prime} Q_{1}{ }^{\prime}\right)\left(1+k_{\nu} Q_{1}{ }^{\prime}\right)^{-1}\left(1+k_{\nu} Q_{2}{ }^{\prime}\right)^{-1} \\
= & \left(1+k_{\nu} Q_{1}{ }^{\prime}\right)^{-1} k_{\nu}{ }^{2}\left(Q_{1}{ }^{\prime} Q_{2}{ }^{\prime}-Q_{2}{ }^{\prime} Q_{1}{ }^{\prime}\right)\left(1+k_{\nu} Q_{2}{ }^{\prime}\right)^{-1}\left(1+k_{\nu} Q_{1}{ }^{\prime}\right)^{-1} \\
& +\left(1+k_{\nu} Q_{1}{ }^{\prime}\right)^{-1} k_{\nu}{ }^{2}\left(Q_{1}{ }^{\prime} Q_{2}{ }^{\prime}-Q_{2}{ }^{\prime} Q_{1}{ }^{\prime}\right)\left\{\left(1+k_{\nu} Q_{1}{ }^{-1}\left(1+k_{\nu} Q_{2}\right)^{-1}\right.\right. \\
= & K_{\nu \nu} \cdot\left(1+k_{\nu} Q_{1}{ }^{\prime}\right)^{-1}+K_{\nu \nu} \cdot P_{\nu \nu}
\end{aligned}
$$

where we used (6.9) to obtain the last identity. Thus by using Lemma 3.1

$$
\left\|P_{\nu}\right\| \leqq\left\|P_{\nu \nu}\right\| \leqq\left\|K_{\nu \nu}\right\|+\left\|K_{\nu \nu}\right\| \cdot\left\|P_{\nu \nu}\right\| .
$$

Hence

$$
\left\|P_{\nu}\right\| \leqq\left\|P_{\nu \nu}\right\| \leqq\left\|K_{\nu \nu}\right\| /\left(1-\left\|K_{\nu v}\right\|\right) .
$$

The estimation of the norm of the second term in (6.8) requires some special care• We use $P_{\nu}{ }^{\prime}$ and $P_{v m}$ defined by

$$
\begin{aligned}
& P_{\nu}{ }^{\prime} \equiv\left(k_{m} / k_{\nu}\right)\left\{\left(1+k_{\nu} Q_{1}{ }^{\prime}\right)^{-1}\left(1+k_{\nu} Q_{2}{ }^{\prime}\right)^{-1}\right. \\
& \left.-\left(1+k_{\nu} Q_{2}{ }^{\prime}\right)^{-1}\left(1+k_{\nu} Q_{1}{ }^{\prime}\right)^{-1}\right\}\left(1+k_{m} Q_{2}{ }^{\prime}\right)^{-1} \\
& =\left(1+k_{\nu} Q_{2}{ }^{\prime}\right)^{-1}\left\{\left(1+k_{\nu} Q_{1}{ }^{\prime}\right)^{-1} k_{\nu} k_{m}\left(Q_{1}{ }^{\prime} Q_{2}{ }^{\prime}-Q_{2}{ }^{\prime} Q_{1}{ }^{\prime}\right)\left(1+k_{\nu} Q_{1}{ }^{\prime}\right)^{-1}\right. \\
& \left.\times\left(1+k_{m} Q_{2}{ }^{\prime}\right)^{-1}\right\}\left(1+k_{\nu} Q_{2}{ }^{\prime}\right)^{-1} \\
& \equiv\left(1+k_{\nu} Q_{2}^{\prime}\right)^{-1} \cdot P_{\nu m}\left(1+k_{\nu} Q_{2}^{\prime}\right)^{-1} .
\end{aligned}
$$

$P_{\nu m}$ can now be treated as $P_{\nu \nu}$ above. We get

$$
\left\|P_{\nu}^{\prime}\right\| \leqq\left\|P_{\nu m}\right\| \leqq\left\|K_{v m}\right\| /\left(1-\left\|K_{\nu m}\right\|\right) .
$$

To finish our proof of (6.2) we use the definition of $R_{\nu}$ and remember that

$$
k_{\nu}<k_{m}, \quad \nu=1,2, \cdots, m-1 .
$$

The proof of (6.3) is quite similar. Using the definition of $R_{\mu}{ }^{\prime}$ and Lemma 3.1 we get

$$
\left\|R_{\mu}{ }^{\prime}\right\| \leqq \sum_{\gamma=1}^{\mu}\left\|T_{\gamma 1} T_{\mu+1,2}-T_{\mu+1,2} T_{\gamma 1}\right\| .
$$

We can treat these terms almost exactly as we did the terms in $R_{v}$ after having observed that

$$
\begin{aligned}
T_{\gamma 1} T_{\mu+1,2}- & T_{\mu+1,2} T_{\gamma 1} \\
& =4\left\{\left(1+k_{\gamma} Q_{1}^{\prime}\right)^{-1}\left(1+k_{\mu+1} Q_{2}^{\prime}\right)^{-1}-\left(1+k_{\mu+1} Q_{2}^{\prime}\right)^{-1}\left(1+k_{\gamma} Q_{1}^{\prime}\right)^{-1}\right\} .
\end{aligned}
$$

This finishes the proof of Lemma 6.1 and thus also the proof of Theorem 1.

We now indicate how to modify the proof above to get a proof of Theorem 2 . Our first modification consists in not carrying out the last similarity transformation in Section 2. We instead define our remainder as

$$
R^{\prime}=\prod_{1}^{m}\left(1+k_{\nu} Q_{2}{ }^{\prime}\right)^{-1}\left(1-k_{\nu} Q_{1}{ }^{\prime}\right)\left(1+k_{\nu} Q_{1}{ }^{\prime}\right)^{-1}\left(1-k_{\nu} Q_{2}{ }^{\prime}\right)-\prod_{1}^{m} T_{\nu 1} \prod_{1}^{m} T_{\nu 2} .
$$


By carrying out an analysis of this remainder analogous to that of $R$ in the proof of Theorem 1, we can prove a lemma corresponding to Lemma 6.1. The main difference in these two lemmas is that the estimate of $\left\|R^{\prime}\right\|$ will contain terms proportional to $\left\|K_{m m}\right\|$. However as the corollary to Lemma 5.1 shows us this is not dangerous if $a_{1}=a_{2}$.

To finish our proof we only have to note that

$$
\begin{aligned}
b \epsilon_{m+1} & =b \prod_{1}^{m}\left(\rho_{\nu}+h^{2} Q_{2}\right)^{-1}\left(\rho_{\nu}+h^{2} Q_{1}\right)\left(\rho_{\nu}+h^{2} Q_{1}\right)^{-1}\left(\rho_{\nu}-h^{2} Q_{2}\right) \epsilon_{1} \\
& =\prod_{1}^{m}\left(1+k_{\nu} Q_{2}{ }^{\prime}\right)^{-1}\left(1-k_{\nu} Q_{1}{ }^{\prime}\right)\left(1+k_{\nu} Q_{1}{ }^{\prime}\right)^{-1}\left(1-k_{\nu} Q_{2}{ }^{\prime}\right) b \epsilon_{1} \\
& =\prod_{1}^{m} T_{\nu 1} \prod_{1}^{m} T_{\nu 2} b \epsilon_{1}+R^{\prime} b \epsilon_{1} .
\end{aligned}
$$

Courant Institute of Mathematical Sciences

New York University

New York, New York 10012

1. G. Birkhoff \& R. S. VARGA, "Implicit alternating direction methods," Trans. Amer. Math. Soc., v. 92, 1959, pp. 13-24. MR $21 \# 4549$.

2. G. BIRKHOFF, R. S. VARGA \& D. M. YOUnG, JR., Alternating-Direction-Implicit Methods, Advances in Computers, Vol. 3, Academic Press, New York, 1962.

3. D. W. Peaceman \& H. H. RAChFord, JR., "The numerical solution of parabolic and elliptic differential equations," J. Soc. Indust. Appl. Math., v. 3, 1955, pp. 28-41. MR 17, 196.

4. C. PEARCY, "On the convergence of alternating direction procedures," Numer. Math., v. 4, 1962, pp. 172-176. MR $26 \# 3206$.

5. R. S. VARGa, Matrix Iterative Analysis, Prentice-Hall, Englewood Cliffs, N. J., 1962. MR $28 \# 1725$.

6. E. L. W AChSPREss, Iterative Solution of Elliptic Systems and Applications to the Neutron Diffusion Equations of Reactor Physics, Prentice-Hall, Englewood Cliffs, N. J., 1966. 